about $2.5 \%$. These calculations suggest that the failure rate might be about $2 \%$ higher with dose 4 than with dose 1 . The observed difference (about $1.5 \%$ ) was in reasonable agreement.

There was evidence of an association between transplacental haemorrhages of $4 \mathrm{ml}$ or more and failures with dose 4 . Twelve women had an estimated transplacental haemorrhage of $4 \mathrm{ml}$ or more after a first pregnancy, and there were three additional women with a transplacental haemorrhage of this extent who were excluded from the trial. If it is assumed that these three women would, if they had been included, have distributed themselves at random among the dose groups then there would have been only about one additional woman with a transplacental haemorrhage of $4 \mathrm{ml}$ or more treated with dose 4. It seems evident that this could not have had any substantial effect upon the results.

The faot that the observed failure rate with a dose of 200$300 \mu \mathrm{g}$ anti-D seemed to be about $1-2 \%$ by the end of a second D-positive pregnancy, as shown by the present trial and by several other series (for example, Eklund and Nevanlinna, 1973), suggests that many apparent failures of treatment when anti-D is given at the time of delivery are due to the occurrence of $\mathrm{Rh}$ sensitization before delivery.

Unless "failures" are carefully defined estimates of the failure rate are bound to vary from one series to another. For example, in the present series women whose serum contained anti-D at the end of their first pregnancy were excluded. No accurate estimate is available of the number of such women excluded but published data suggest that the figure would be about 0.5\% (Woodrow, 1970; Eklund and Nevanlinna, 1973). On the same reasoning it may be assumed that about $0.5 \%$ of women develop anti-D at the end of the second pregnancy as a result of primary immunization in that pregnancy. Such women will falsely be included as failures of treatment.

The criterion of "serologically detectable anti-D" must also affect the reponted failure rate. In the present series only women who developed a positive I.A.G.T. result were counted as failures. The five women with anti-D detectable only with enzyme-treated cells at the end of their second pregnancy were not counted as failures. Nevertheless, even if they had been included the overall failure rate would have risen by less than $1 \%$.

In the United Kingdom and in a few other countries a dose of $100 \mu \mathrm{g}$ anti-D has for some time been used for routine administration to unimmunized D-negative women recently delivered of a D-positive infant. Our results support the contention that this dose has a success rate which is not appreciably different from that observed with a dose of 200$300 \mu \mathrm{g}$. Whatever standard dose is adopted it is desirable to perform a screening test to detect large transplacental haemorrhages because it is likely that in such cases the risk of $\mathrm{Rh}$ immunization can be reduced by giving an appropriately increased dose of anti-D.

Requests for reprints to: M.R.C. Experimental Haematology Unit, St. Mary's Hospital Medical School, London W2 1PG.

\section{References}

Betke, K., and Kleihauer, E. (1958). Blut, 4, 241.

Clarke, C. A. (1967). British Medical Fournal, 4, 7

Eklund, J., and Nevanlinna, H. R. (1973). Fournal of Medical Genetics, 10 $1-7$.

Hughes-Jones, N. C., and Stevenson, Mary. (1968). Vox Sanguinis, 14, 401.
Mollison, P. L. (1972). Blood Transfusion in Clinical Medicine, 5th edn., Oxford, Blackwell Scientific Publications.

Smith, G. N., Mollison, D. P., Griffiths, B., and Mollison, P. L. (1972) Lancet, 1, 1208.

Tee, D. E. H., and Watkins, J. (1967). British Medical fournal, 4, 210

Wallace, J. (1971). Personal communication.

World Health Organization. (1971). Prevention of Rh Sensitization. Technical Report Series, No. 468, p. 29, Geneva, W.H.O.

Woodrow, J. C. (1970). Series Haematologica, 3, 3.

\title{
Hepatic Disorders Associated with Liver/Kidney Microsomal Antibodies
}

\author{
M. G. M. SMITH, ROGER WILLIAMS, GEOFFREY WALKER, M. RIZZETTO, \\ DEBORAH DONIACH
}

British Medical fournal, 1974, 2, 80-84

\section{Summary}

A study of the clinical associations of a recently defined tissue autoantibody, the liver/kidney microsomal (L.K.M.) antibody, showed that out of 33 patients 26 had clinical liver disease. Fifteen of the patients had active chronic hepatitis and there were seven cases of acute hepatitis, precipitated by presumed virus $A$ infection in three instances and by drug hypersensitivity in the other four. The remaining cases with liver disease included two with subclinical hepatitis and two with

Liver Unit, King's College Hospital Medical School, London SE5 8RX M. G. M. SMITH, M.B., M.R.C.P., Senior Registrar ROGER WILLIAMS, M.D., F.R.C P., Director

St. Mary's Hospital, London W2 1 NY

GEOFFREY WALKER, M.D., M.R.C.P., Consultant Gastroenterologist

Middlesex Hospital and Medical School, London W2 7PN

M. RIZZETTO, M.D., (Turin), Research Fellow, (Present address:

Gastroenterology Department, Mauriziano Hospital, Turin, Italy) DEBORAH DONIACH, M.D., F.R.C.P., Consultant Immunopathologist hepatocellular carcinoma. Evidence is presented that the patients with active chronic hepatitis may represent a distinct subgroup of the disease with a young mean age, an even male to female ratio, and a striking lack of other nonorgan-specific autoantibodies-that is, antinuclear and smooth muscle-which are usually present in the other autoimmune variant of the disease.

\section{Introduction}

Immunofluorescent tests are helpful in the differential diagnosis of centain chronic liver diseases. The most useful markers have been the antinuclear (A.N.A.) and smooth muscle (S.M.A.) antibodies (Holborow, 1972), present in some cases of active chronic hepatitis, and the mitochondrial antibodies (A.M.A.), which are found in almost all patients with primary biliary cirrhosis (Walker et al., 1965; Klatskin and Kantor, 1972; Sherlock and Scheuer, 1973). Mitochondrial antibodies react with all organs and all types of mitochondria and the immunofluorescent pattern is now well characterized (Doniach, 1972). The antigen has been localized to a lipoprotein in the inner mitochondrial membranes (Berg et al., 1969; BenYoseph et al., 1974). Binding of immunoglobulins 
from patients with primary biliary cirrhosis to these membranes has also been shown by immunoelectronmicroscopy with peroxidase conjugates (Bianchi et al., 1973).

In the past four years other immunofluorescent patterns closely resembling A.M.A. have been recognized. Of particular interest for liver disorders is an antibody which has now been named the liver/kidney microsomal (L.K.M.) antibody as it reacts mainly with hepatocytes and proximal renal tubules. At first it was thought to represent a variant of A.M.A. since it occurred mostly in liver patients and certain sera fixed complement with mitchondrial preparations (Doniach and Walker, 1972). This antibody has now been further characterized, however, and the antigen localized to microsomal membranes (Rizzetto et al., 1973). Separation of A.M.A. and L.K.M. antibodies was finally achieved by quantitative complement fixation studies and absorption of the immunofluorescence with purified subcellular fractions. Localization to rough endoplasmic reticulum was also confirmed by immunoelectronmicroscopy (Rizzetto et al., 1974).

L.K.M. antibodies are uncommon and we describe here the clinical conditions in which they were found.

\section{Patients and Methods}

A total of 33 patients (16 male, 17 female) were found to have
L.K.M. antibodies in the serum. This antibody was detected in $0.12 \%$ of the patients whose serum was sent to the laboratory for testing after December 1972. Most of the 33 were retested at intervals over periods up to four years. The results were persistently positive except in three patients in whom the antibody could no longer be detected after intervals of two months to one year. Sera could be kept at $-20^{\circ} \mathrm{C}$ for several years without loss of antibody activity.

Sera were screened at $1 / 10$ dilutions by indirect immunofluorescence with polyvalent anti- $\gamma$ FITC conjugates. To distinguish L.K.M. from mitochondrial antibodies it was essential to include both renal cortex and medulla, which necessitated changing from human to rat kidney. A.M.A. stains all renal tubules with maximum fluorescence on distal tubule and ascending loop of Henle whereas L.K.M. antibody reacts mostly with the $P_{3}$ portion of proximal tubules and gives no staining of the renal medulla. On liver the staining panterns of these two antibodies are difficult to distinguish, while thyroid and stomach are awkward substrates owing to the organ-specific thyroid and gastric parietal cell antibodies which are sometimes present together with L.K.M. in liver patients. All other antibody tests were as described in the W.H.O. manual for autoimmune serology (Roitt and Doniach, 1969). Serum immunoglobulin (Ig) levels were measured by single radial immunodiffusion (Mancini et al., 1965). Hepatitis $B$ antigen (HBAg) and antibodies were detected by radioimmunoassay and by haemagglutination inhibition tests.

Clinical and Serological Data on 33 Patients with L.K.M. Antibodies

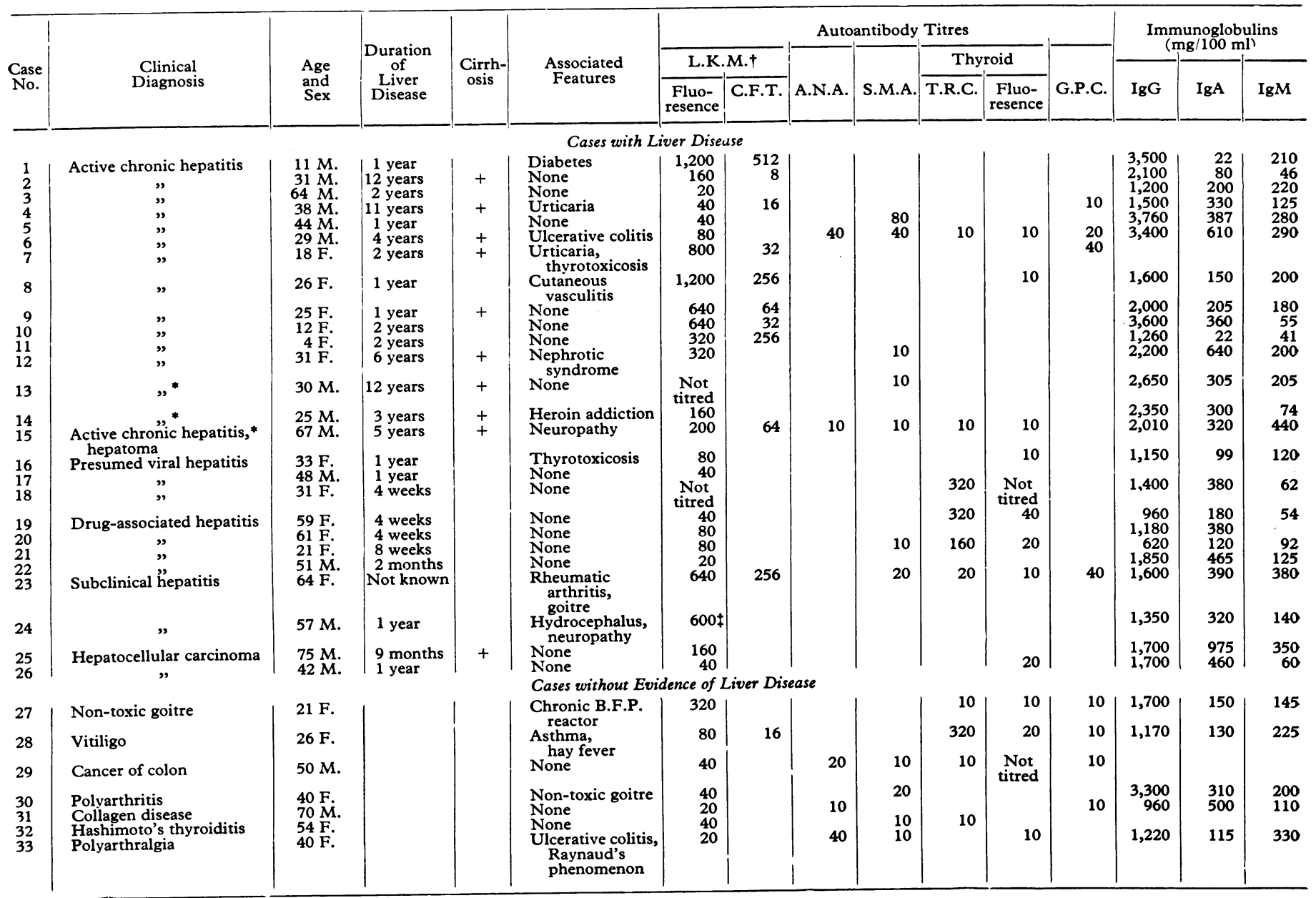

B.F.P. $=$ Biological false positive test for syphilis.

C.F.T. = Complement fixation test.

T.R.C. = Tanned Red Cell agglutination test for thyroglobulin antibodies.

G.P.C. = Gastric parietal cell.

* On repeated testing $\mathrm{HB}+\mathrm{g}$ was positive in serum.

+ Maximum L.K.M. fluoresence titre is given where repeated specimens were tested.

† Monoclonal IgGs Kappa. 


\section{Results}

Clinical and serological data are shown in the table. Twentysix patients had confirmed liver disease and L.K.M. antibodies were also found in seven patients who presented with nonhepatic conditions and whose liver funotion tests gave normal results.

\section{LIVER DISORDERS}

\section{Active Chronic Hepatitis}

The 15 patients with active hepatitis ( 9 male, 6 female) ranged in age from 4 to 67 years (mean 30 years). The known age of onset of the chronic liver condition ranged from 2 to 62 years (mean 26 years) and the duration of the illness ranged from one to 12 years (mean five years). Twelve cases presented with jaundice and two of the three remaining patients had had episodes of jaundice 10 and 20 years previously. Cirrhosis was present at the time of initial diagnosis in two patients and was seen to develop during follow-up in seven funther cases while three others showed progressive liver damage and heavy fibrosis. All but two of the 15 patients had received corticosteroid therapy, alone or in combination with azathioprine. Two patients who developed cirrhosis died four and six years after the onset of symptoms, one with a hepatocellular carcinoma. The three patients with positive tests for HBAg in the serum -the only such cases in the complete series-were all men and two had special reasons for contracting the virus (one was a chronic heroin addict and the other had had homosexual contacts). Associated clinical disonders included diabetes mellitus in one patient, severe chronic urticaria in two, thyrotoxicosis in one, colitis in one, cutaneous vasculitis in one, and peripheral neuropathy in one.

Microsomal fluorescence titres varied between 20 and 1,200 and were 160 or higher in two-thirds of the active chronic hepatitis cases. Complement fixation with liver microsomes was positive in nine cases; with titres up to 512 . In some patients tested repeatedly there were marked fluctuations. Rising titres were observed during aotive progression of the disease (cases 8 and 10) and a decrease occurred when the hepatitis became quiescent, as seen in case 1 , or when an established cirrhosis became inactive, as in case 7. Other antibodies were found in trace amounts. Only one patient (case 6) had significant A.N.A.; L.E. cells were shown to be present at the onset of the disease, with A.N.A. of 40 on one occasion, but the A.N.A. was praotically negative on subsequent occasions. Four patients had traces of S.M.A., one reaching a titre of 80 , and five had low titres of organ-specific thyroid or gastric parietal cell antibodies. Serum IgG levels were above $3,000 \mathrm{mg} / 100 \mathrm{ml}$ in four patients, three of whom had not developed cirrhosis. IgA showed no strikingly high values and was abnormally low $(80,22,22 \mathrm{mg} / 100 \mathrm{ml})$ in three patients. IgM values were significantly raised in two patients, both with cirrhosis.

Comparison of Patients with L.K.M.-positive and L.K.M.negative Active Chronic Hepatitis.-To determine whether patients with L.K.M.-positive aotive chronic hepatitis could be distinguished as a group from other patients with this disease statistical comparisons were made with a series of 89 patients with active chronic hepatitis seen at King's College Hospital in the last five years (Reed et al., 1973). The latter included 13 patients with persistent hepatitis $B$ antigenaemia. The mean age of 26 years at the onset of liver disease in the L.K.M.positive group was lower than that of the L.K.M.-negative patients (mean 41 years; range 9 days -77 years; $P<0.005$ ). There was a slight male preponderance among the L.K.M.positive patients $-60 \%$ compared with $34 \%$ in the L.K.M. negative patients-but this was not statistically significant.
The mean duration of liver disease was about five years in both groups and a similar percentage of each presented with an acute illness resembling viral hepatitis. Non-organ specific S.M.A. and A.N.A. were found much less often overall among the L.KM.-positive patients; only five of the 15 showed any trace of these antibodies compared to 76 of the L.K.M.negative subjects. When antibody titres of 80 or more only were considered and related to sex for the comparison, however, this clear-cut difference was restrioted to the female patients. None of the six female patients in the L.K.M.positive group had more than trace amounts of A.N.A. or S.M.A. compared to 31 out of 58 L.K.M.-negative female patients $(P=0.014)$. In contrast, two of the nine L.K.M.positive men had high titre antibodies compared to 10 out of 31 L.K.M.-negative male patients (N.S. $P=0.28$ ).

\section{Presumed Acute Viral Hepatitis}

Of three patients (one man and two women) with presumed acute viral hepatitis two had had relapsing jaundice for over a year with consistently negative HBAg. Liver biopsy in the man showed resolving acute hepatitis. One of the women had a past history of thyrotoxicosis. There was no evidence of drug abuse or alcoholism and the hepatitis has since remitted in both cases. The thind case was admitted with acute hepatic necrosis and died of liver failure, with a hepatic weight of $839 \mathrm{~g}$. All three patients in this group had low titres of L.K.M. fluorescence (40-80) and two also had thyroid antibodies. In one patient tested repeatedly (case 16) the L.K.M. antibodies remained positive at the same titre throughout a two year follow-up period.

\section{Drug-associated Hepatitis}

Three women and one man had drug-associated hepatitis. The women all developed jaundice after a repeated halothane anaesthetic. In case 19 the patient died with acute necrosis within a month of onset (liver weight $580 \mathrm{~g}$ ). In case 20 there was a non-icteric hepatitis (maximum bilirubin $1.3 \mathrm{mg} / 100$ $\mathrm{ml}$; aspartate aminotransferase (SGOT) $800 \mathrm{IU} / 1$.) characterized on liver biopsy by centrizonal necrosis, a granulomatous reaction, and an eosinophil periportal infiltrate. Clinically, a high fever persisted for over a week. The patient made a full recovery within one month. In case 21 the patient developed hepatic necrosis and deep coma seven days after her second halothane anaesthetic for a submucous nasal resection, and at the time of writing she was recovering. The male patient had been taking methyldopa for mild hypentension for two weeks when he developed anorexia, high fever, and subsequent jaundice. His bilirubin rose to a maximum of $3.6 \mathrm{mg} / 100 \mathrm{ml}$ and SGOT to $55 \mathrm{IU} / 1$. There was a mild peripheral eosinophilia of $600 / \mathrm{mm}^{3}$. Liver biopsy showed modest portal inflammation with mainly lymphocytic infiltrate and centrizonal swelling of liver cells. An oral cholecystogram gave normal results. The patient made a slow recovery and the results of liver function tests returned to normal on stopping methyldopa.

Serologically these four patients showed L.K.M. fluorescence to titres of 20-80, decreasing as the condition improved in two cases. Two of the female patients also had thyroid antibodies.

\section{Subclinical Hepatitis}

There were two cases of subclinical hepatitis. A 64-year-old woman wih a 22-year history of seropositive rheumatoid anthritis needing long-term steroid treatment was found to have hepatomegaly with a slight increase in serum alkaline phosphatase (16 K.A. units). She also had a non-toxic goitre and 
was known to have had a false positive reaction for syphilis for some years. No biopsy was available. The second patient was a 57-year-old man who presented with peripheral neuropathy, papilloedema, and intermittent coma. Extensive investigations had shown raised cerebrospinal fluid pressure and protein content of $110 \mathrm{mg} / 100 \mathrm{ml}$, and air studies showed dilatation of lateral ventricles. There had been an episode of painless jaundice six years earlier. Liver biopsy showed expansion of portal tracts with mononuclear cells and there was patchy liver cell necrosis. No amyloid was seen in rectal and liver biopsy material. On the suspicion of collagenosis he was treated with corticostenoids but showed no response. He died after one and a half years of illness. No myeloma or other malignancy was found at necropsy and the central nervous system showed advanced atrophy of neurons with demyelination. On serum electrophoresis there was a monoclonal spike identified as $\mathrm{IgG}_{3}$ kappa and this contained all the L.K.M. fluorescence activity, which could be shown to a titre of 600 .

\section{Primary Hepatocellular Carcinoma}

The first of the two patients with primary hepatocellular carcinoma was a 75-year-old man who presented with haematemesis, preceded by nine months' vague ill-health. At necropsy a large hepatocellular carcinoma was found spreading into surrounding structures, the sections also showing an underlying micronodular cirrhosis. L.K.M. antibodies were found to a titre of 160 and the serum immunoglobulins showed raised IgA and IgM values. The second patient, a man aged 42, had an anaplastic liver cell cancer with no evidence of underlying cirrhosis at laparotomy. He showed L.K.M. fluorescence to $1 / 40$ and thyroid to $1 / 20$ repeatedly until shortly before he died one year after the onset of symptoms.

\section{NON-HEPATIC DISEASE}

In five of the seven patients whose liver function tests gave normal results the L.K.M. antibody was detected at intervals up to two years. One was a healthy girl with a biological false positive reaotion for syphilis and a non-toxic goitre who had L.K.M. fluorescence to a titre of 320 , the antibodies being of IgM class. Another was a 28-year-old woman with extensive chronic vitiligo; a palpable thyroid; longstanding history of allergy, including skin hypersensitivities; and hay fever. Her mother had myxoedema and three other relatives had vitiligo. Two patients with low-titre L.K.M. antibodies had polyarthritis and evidence of collagen disease and one man allegedly had carcinoma of the colon. In the remaining two patients, with longstanding Hashimoto's disease and polyarthralgia respectively, L.K.M. antibodies were found on one occasion only and specimens taken nine to 12 months later were negative.

\section{Discussion}

The separate identity of the L.K.M. antibody and its reaction with microsomal membranes of the rough endoplasmic reticulum can now be accepted on the basis of quantitative complement fixation, absorption of immunofluorescence with appropriate subcellular fractions, and immunoeleotronmicroscopy findings with peroxidase-coupled antibodies. This autoantibody seems to be far less common than A.M.A. Whereas 158 A.M.A.-positive sera were detected among 7,500 new sera tested in 1973 only nine with L.K.M. were found on intensive search. It is striking that 26 out of the 33 sera containing these antibodies were derived from patients with overt liver disease, no less than 15 of whom had active chronic hepatitis.

Active chronic hepatitis may have several underlying causes, and though in most cases the aetiological or initiating factors have not been identified two subgroups of the syndrome have been defined. Persistent hepatitis B antigenaemia is associated with some cases of active chronic hepatitis in which there is a male predominance (Reed et al., 1973), while centain drugsfor example, oxyphenisatin (Reynolds et al, 1971; Gjone et al., 1972)-may produce a chronic hepatitis associated with the appearance of A.N.A. and S.M.A. in which both the disease and the antibodies regress when the drug is discontinued. A more permanent and intense autoimmunization may be observed in relation to "lupoid" hepatitis, where A.N.A. and S.M.A. are the conspicuous markers, and also in a small subgroup-mainly of middle-aged women showing high titres of A.M.A. with obstruotive features and raised serum alkaline phosphatase values-which may overlap with the primary biliary cirrhosis group.

The finding of L.K.M. antibodies perhaps makes it possible to single out another subgroup of active chronic hepatitis cases which show some differences from the other groups. Striking features were the lower frequency of A.N.A. and S.M.A., the higher propontion of males, the younger age group with a relatively high incidence in children, and the defined icteric onset of the illness in nearly all of the cases studied so far. Comparison with a large group of patients with active chronic hepatitis without L.K.M. antibodies showed maximum serological differences in female patients, suggesting that our group was distinct from lupoid hepatitis. One other feature of uncentain significance was the finding of low IgA levels in three of the cases, though this high incidence does not provide statistical distinction from the L.K.M.-negative patients.

With regard to the difficulty in distinguishing between mitochondrial and L.K.M. fluorescence, previous series of active chronic hepatitis always contained a proportion of sera giving fluorescence patterns resembling A.M.A. which could not be interpreted in earlier studies. Conversely, it may be asked whether L.K.M. antibodies are present in some patients with primary biliary cirrhosis in addition to or instead of A.M.A. This is particularly relevant in view of the unexplained positive results of complement fixation studies with microcomal preparations observed in over $25 \%$ of primary biliary cirrhosis cases. (Doniach et al., 1966). No firm answer can be given on this point. Mitochondrial antibodies react with all organs wheras L.K.M. staining is almost confined to liver and kidney. Despite looking very carefully for the two immunofluorescent patterns in 50 selected primary biliary cirrhosis sera it was impossible to see concomitant L.K.M. fluorescence. Furthermore, we have no doubt that many tissue antibodies have yet to be identified and are at present poorly understood.

The relation of L.K.M. antibodies to HBAg is at present difficult to define. We have failed to deteot this autoantibody in many cases of viral hepatitis type B, though L.K.M. fluorescence was found in sera from three patients with active chronic hepatitis associated with HBAg. Prolonged followup with repeated antibody tests should be done in druginduced hepatitis cases in order to ascentain whether the L.K.M. antibodies disappear with time, as this would be in favour of their stimulation by the drug hypersensitivity. The same applies to the occasional A.M.A. seen after halothaneassociated jaudice (Rodriguez et al.. 1969: Simpron ot al., 1973). The two cases thought to have subclinical hepatitis were of special interest. The woman with chronic rheumatoid arthritis and biological false positive reaction was in some ways similar to the patients with A.M.A., "collagen" disorders, and subclinical liver disease studied earlier (Walker et al., 1970; Whaley et al. 1970). Some of these patients had fluorescence patterns which were atypical for A.M.A. Similar difficulties of interpretation were found in a series of patients with false positive reactions for syphilis (Doniach et al., 1970). In the male patient with neurological disease and subclinical hepatitis the L.K.M. antibody was confined entirely to a monoclonal band (Florin-Christensen et al., 1974) and the significance of this is unknown. 
In seven patients low titre L.K.M. antibodies were found in the absence of any evidence of liver disease and in two of these their appearance was temporary. All but one of these patients suffered from collagenoses or thyroid autoimmunity. In this context it is interesting that in half of all patients having L.K.M. antibodies, irrespective of diagnosis, thyroid or gastric organ-specific antibodies also occurred. The identity of these antibodies could be shown by selective absorption of the thyroid or gastric immunofluoresence, leaving intact that due to the L.K.M. The L.K.M. antibody with its restricted organ reactivity may represent an intermediate form of autoimmunity between the strict organ specificity of the thyroiditis/gastritis/adrenalitis group of disorders and the complete non-organ specificity of such autoantibodies as A.M.A. and A.N.A.

Studies are in progress to attempt a separation of L.K.M.associated active chronic hepatitis by tissue typing (Mackay and Morris, 1972) and the incidence of high titre viral antibodies, including rubella, measles, and herpes (Triger et al., 1972), from the largest group of active chronic hepatitis patients who show no autoimmunity or $\mathrm{HBAg}$ and from the subgroups associated with high titres of A.N.A. and S.M.A. and those showing A.M.A. in the serum.

We thank Dr. Dudley Tee of the department of experimental pathology at King's College Hospital for the immunoglobulin measurements and Dr. Richard Stern for the statistical analysis. Dr. W. D. Reed kindly made available the comparative data from a series of chronic active hepatitis cases. We also thank Mr. and Mrs. G. Swana for their skilled technical help.

\section{ADDENDUM}

Since this paper was submitted further information on the clinical significance of the L.K.M. antibody has come from Dr. J-C. Homberg, of Paris. He observed this immunofluorescence pattern independently and with other French immunopathologists collected 14 positive cases ( $11 \mathrm{~F}$., $3 \mathrm{M}$.) over the past five years, representing about $0 \cdot 1 \%$ of all sera tested. Ten were from patients with either active chronic hepatitis in young subjects or with unexplained cirrhosis (Homberg et al., 1974).

\section{References}

Ben-Yoseph, Y., Shapira, E., and Doniach, D. (1974). Immunology, 26.

In press.
Berg, P. A., Roitt, I. M., Doniach, D., and Horne, R. W. (1969). Clinical and Experimental Immunology, 4, 511.

Bianchi, F. B., Penfold, P. L., and Roitt, I. M. (1973). British fournal of Experimental Pathology, 54, 652.

Doniach, D. (1972). In Progress in Clinical Immunology, vol. 1, p. 45. New

York, Grune and Stratton.
Doniach, D., Delhanty, J., Lindqvist, H. J., and Catterall, R. D. (1970). Clinical and Experimental Immunology, 6, 871.

Doniach, D., Lindqvist, H. L., and Berg, P. A. (1966). International Archives of Allergy, 41, 501 .

Doniach, D., and Walker, J. G. (1972). In Progress in Liver Diseases, ed. H. Popper and F. Schaffner, vol. IV. New York, Grune and Stratton.

Florin-Christensen, A., Doniach, D., Walker, J. G., and Kocen, R. S. (1974).

In preparation.
Gjone, E., Blomhoff, J. P., Ritland, S., Elgio, K., and Husby, G. (1972). Scandinavian fournal of Gastroenterology, $7,395$.

Holborow, E. J. (1972). Proceedings of the Royal Society of Medicine, 65, 481.

Homberg, J. C., Micouin, C. Peltier, A., Salmon, C., and Caroli, J., (1974). Medicine et Chirurgie Digestives. In press.

Klatskin, G., and Kantor, F. S. (1972). Annals of Internal Medicine, 77, 533.

Mackay, I. R., and Morris, P. J. (1972). Lancet, 2, 793.

Mancini, G., Carbonara, A. O., and Heremans, J. F. (1965). International Fournal of Immunochemistry, 2, 235.

Reed, W. D., et al. (1973). Lancet, 2, 690.

Reynolds, T. B., Peters, R. L., and Yamada, S. (1971). New England fournal of Medicine, 285, 813 .

Rizzetto, M., Bianchi, F. B., and Doniach D. (1974). Immunology, 26.

In press.
Rizzetto, Mwana, G., and Doniach, D. (1973). Clinical and Experimental Immunology, 15, 331 .

Rodriguez, M., Paronetto, F., Schaffner, F., and Popper, H. (1969). fournal of the American Medical Association, 208, 148.

Roitt, I. M., and Doniach, D. (1969). W.H.O. Manual of Autoimmune Serology, Geneva, World Health Organization.

Sherlock, S., and Scheuer, P. J. (1973). New England fournal of Medicine, 289, 674 .

Simpson, B. R., Strunin, L., and Walton, B. (1973). Proceedings of the Royal Society of Medicine, 66, 56.

Triger, D. R., Kurtz, J. B., MacCallum, F. O., and Wright, R. (1972). Lancet, 1, 665.

Walker, J. G., Doniach, D., and Doniach, I. (1970). Quarterly fournal of Medicine, 39, 31 . Walker, J. G., Doniach, D., Roitt, I. M., and Sherlock, S. (1965). Lancet, 1,

Whaley, K., Goudie, R. B., Williamson, J., Nuki, G., Dick, W. C., and Buchanan, W. W. (1970). Lancet, 1, 861 .

\title{
Haemolytic-Uraemic Syndrome in Typhoid Fever
}

\author{
N. M. BAKER, A. E. MILLS, I. RACHMAN, J. E. P. THOMAS
}

British Medical fournal, 1974, 2, 84-87

\section{Summary}

Among 48 patients with a typhoid infection 6 (12.5\%) developed the haemolytic-uraemic syndrome. Neither glucose-6phosphate dehydrogenase deficiency nor therapy with chloramphenicol could be incriminated as the causal factor. Evidence presented here suggests that the mechanism is localized intravascular coagulation.

The presence of leucocytosis in typhoid fever suggests a complication and should alert one to the possibility of the haemolytic-uraemic syndrome. Furthermore, in our area typhoid should be suspected as a cause in any patient presenting with acute renal failure.

Division of Medicine, Mpilo Central Hospital, Bulawayo, Rhodesia N. M. BAKER, M.B., M.R.C.P., Physician

A. E. MILLS, M.B., D.PATH., Pathologist

I. RACHMAN, M.B., M.R.C.P., Physician

\section{Introduction}

Haemolysis and renal failure have been regarded as rare complications of typhoid fever. Retief and Hofmeyr (1965) found fewer than 40 cases of haemolytic anaemia associated with typhoid noted in the literature. Since then there have been further reports, usually of single cases. That this complication might be more common was suggested when Lwanga and Wing (1970), in a two-year retrospective study, found that of 130 patients with typhoid $7 \%$ had evidence of haemolysis, but that was in areas where glucose-6-phosphate dehydrogenase (G-6-PD) deficiency was common. Hersko and Vardy (1967) described haemolysis in five children, all with G-6-PD deficiency. Huckstep (1962) mentioned an incidence of haemolytic anaemia of $2 \%$ and of "nephrotyphoid" of $1 \%$. Gulati et al. (1968) reported two cases of nephritis among 98 patients, and Wicks et al. (1971) reported renal complications in six out of 265 patients.

Lwanga and Wing (1970) claimed the first reported case of acute oliguric renal failure after intravascular haemolysis in typhoid fever. That was in a patient with G-6-PD deficiency. Though Allen (1969) reported one case with consumption co- 